\title{
Inter-site Coulomb interaction and Heisenberg exchange
}

\author{
R. Eder, J. van den Brink, and G. A. Sawatzky \\ Department of Applied and Solid State Physics, University of Groningen, \\ 9747AG Groningen, The Netherlands
}

(today)

\begin{abstract}
Based on exact diagonalization results for small clusters we discuss the effect of inter-site Coulomb repulsion in Mott-Hubbard or charge transfers insulators. Whereas the exchange constant $J$ for direct exchange is enhanced by inter-site Coulomb interaction, that for superexchange is suppressed. The enhancement of $J$ in the single-band models holds up to the critical value for the charge density wave (CDW) instability, thus opening the way for large values of $J$. Single-band Hubbard models with sufficiently strong inter-site repulsion to be near a CDW instability thus may provide physical realizations of $t-J$-like models with the 'unphysical' parameter ratio $J / t \approx 1$.
\end{abstract}

74.20.-Z, 75.10.Jm, 75.50.Ee

A frequently used approach to discuss the strongcoupling limit of Hubbard-type models is the perturbation expansion in $t / U$ [1,2], which leads to an effective Hamiltonian operating in the subspace of states without double occupancies, the well-known $t-J$ model. By virtue of its perturbational derivation, the relation $J=4 t^{2} / U$ holds, so that it seems that a necessary condition for the validity of $t-J$-like models is the relation $J / t=4(t / U) \ll 1$. In this work we show that this is not necessarily the case; rather, Hubbard models with an additional inter-site repulsion which is sufficiently strong to bring the system close to a charge-density-wave instability may provide 'physical' realizations of $t-J$ models with $J / t \approx 1$. We also show that the situation is reversed in a charge-transfer insulator, where inter-site Coulomb repulsion on the contrary suppresses the superexchange. To begin with, it is advantageous to develop a simple picture of a correlated insulator. We restrict ourselves to $1 D$ systems in all what follows, the main reason being that exact diagonalization for reasonably large $2 D$ clusters of the single or even two-band Hubbard model is not feasible. We expect, however, that the physical considerations outlined below retain their validity in any dimension. We consider the single band Hubbard model

$H=-t \sum_{i, \sigma}\left(c_{i, \sigma}^{\dagger} c_{i+1, \sigma}+H . c\right)+U \sum_{i} n_{i, \uparrow} n_{i, \downarrow}+V \sum_{i} n_{i} n_{i+1}$

where $n_{i, \sigma}=c_{i, \sigma}^{\dagger} c_{i, \sigma}, n_{i}=n_{i, \uparrow}+n_{i, \downarrow}$. The model already includes an additional repulsion between electrons on nearest neighbors $\sim V$. We also consider the charge tranfer system

$$
\begin{aligned}
H=-t & \sum_{i, j \in N(i), \sigma}\left(d_{i, \sigma}^{\dagger} c_{j, \sigma}+H . c\right)+U \sum_{i} n_{i, \uparrow} n_{i, \downarrow} \\
& -\Delta \sum_{i} n_{i}+V \sum_{i, j \in N(i)} n_{i} n_{j},
\end{aligned}
$$

which consists of strongly correlated ' $d$ '-orbitals separated by uncorrelated ligands $(N(i)$ denotes the two ligand sites neighboring the $d$-site $i$ ). The on-site energy
$-\Delta$ of the $d$-orbitals is taken to be negative, and in addition to the $d$-site Coulomb repulsion $U$ we include an additional Coulomb repulsion $V$ between nearest neighbor $d$ and ligand orbitals.

At 'half-filling' and in the limit $V=0, U \rightarrow \infty$ we have precisely one electron per lattice site for the Hubbard model, and one electron (or hole) per $d$-site in the charge transfer system (see the states labeled ' 0 ' in Figure 1).

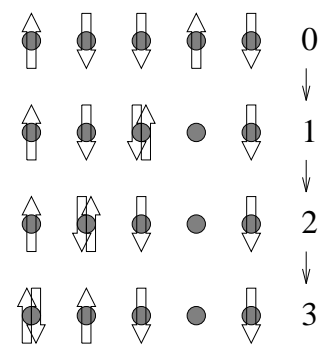

(a)

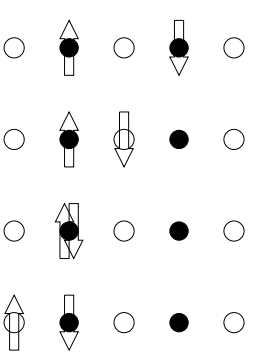

(b)

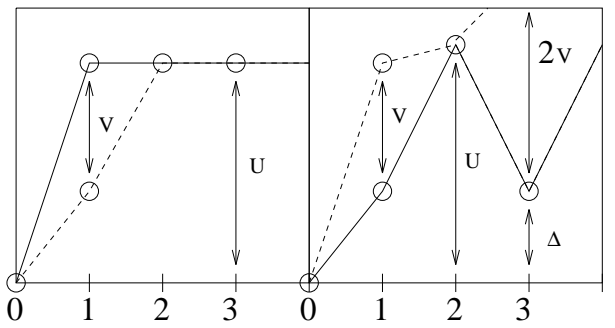

FIG. 1. Charge fluctuations in the single band Hubbard model (a) and the charge transfer system (b). The dark circles in part (b) indicate the 'd' sites, the light ones the ligands. The lower part of the figure gives the energies of the states created by the charge fluctuation with (dashed line) and without (full line) a nearest neighbor repulsion of strength $V$ taking the hopping integral to be 0 .

Switching on the inter-site kinetic energy then will lead to charge fluctuations: in the Hubbard model, an electron can jump to a nearest neighbor, thus creating a 'hole' and a 'double occupancy' on nearest neighbors. The energy 
thereby is increased by the large amount $U$. In subsequent steps hole and the double occupancy can separate even further, but all states generated in this way have the same large Coulomb energy $U$ (see Figure 11). For the case of the charge-transfer system, similar considerations hold, with the energy required to 'drag apart' hole and double occupancy being $\min (\Delta, U)$. By analogy with the problem of a particle moving in a $1 \mathrm{D}$ attractive $\delta$ potential one may therefore expect that the probability to find a hole and a double occupancy at a distance of $n$ lattice spacings decreases rapidly with $n$. The canonical transformation to a Heisenberg or $t-J$ Hamiltonian with only nearest neighbor exchange then obviously is equivalent to assuming that states where hole and double occupancy are more distant than nearest neighbors (nearest neighbor $d$-sites for the case of the charge transfer system) can be completely neglected.

Let us now consider the case $V>0$. For the single-band Hubbard model the energy necessary to create a charge fluctuation on nearest neighbors is lowered to $U-V$ (see Figure 11). The energy to drag apart hole and double occupancy, however, remains unchanged, so that the separation between the two Hubbard bands (which are associated with 'free' propagation of hole or double occupance) stays unaffected [3, 4]. Invoking again the analogy with a particle moving in a $1 \mathrm{D}$ attractive potential one might instead expect the formation of a 'bound state' between a hole and a double occupancy on nearest neighbors, which manifests itself as an excitonic excitation at an energy well below the Hubbard gap in the optical conductivity [4]. The $V$-term therefore does not reduce the Hubbard gap, and in particular does not 'screen' $U$ to turn the correlated insulator into a weakly correlated metal. The question remains, however, whether a transformation to a $t-J$ model with only nearest neighbor exchange still makes sense in the presence of the $V$-term. Obviously, if there is an appreciable probability to have the hole-double occupancy pair on more distant than nearest neighbor sites, longer range spin correlations will become important in the effective low energy Hamiltonian. To address this question we use exact diagonalization of small clusters; we note that for the short-range/high energy processes which mediate the Heisenberg exchange finite size effects probably play only a minor role. We introduce $n_{i, d}=n_{i, \uparrow} n_{i, \downarrow}$ and $n_{i, h}=\left(1-n_{i, \uparrow}\right)\left(1-n_{i, \downarrow}\right)$, i.e., the density operators for doubly occupied and empty sites and study the 'empty-double correlation function'

$$
g(R)=\frac{1}{N} \sum_{i}\left\langle n_{i, h} n_{i+R, d}\right\rangle
$$

(where $N$ denotes the chain length) as a function of the nearest neighbor repulsion $V$. This is shown in Figure 22. Obviously $g(R)$ is peaked at $R=1$, i.e., independently of the value of $V$ 'hole' and 'double occupancy' strongly prefer to be on nearest neighbors. For $R>1$ and not too large $V$ the empty-double correlation immediately levels off to a practically $R$-independent value $g_{\infty}$; this can be understood as the contribution from holes and double occupancies belonging to different 'pairs': since the
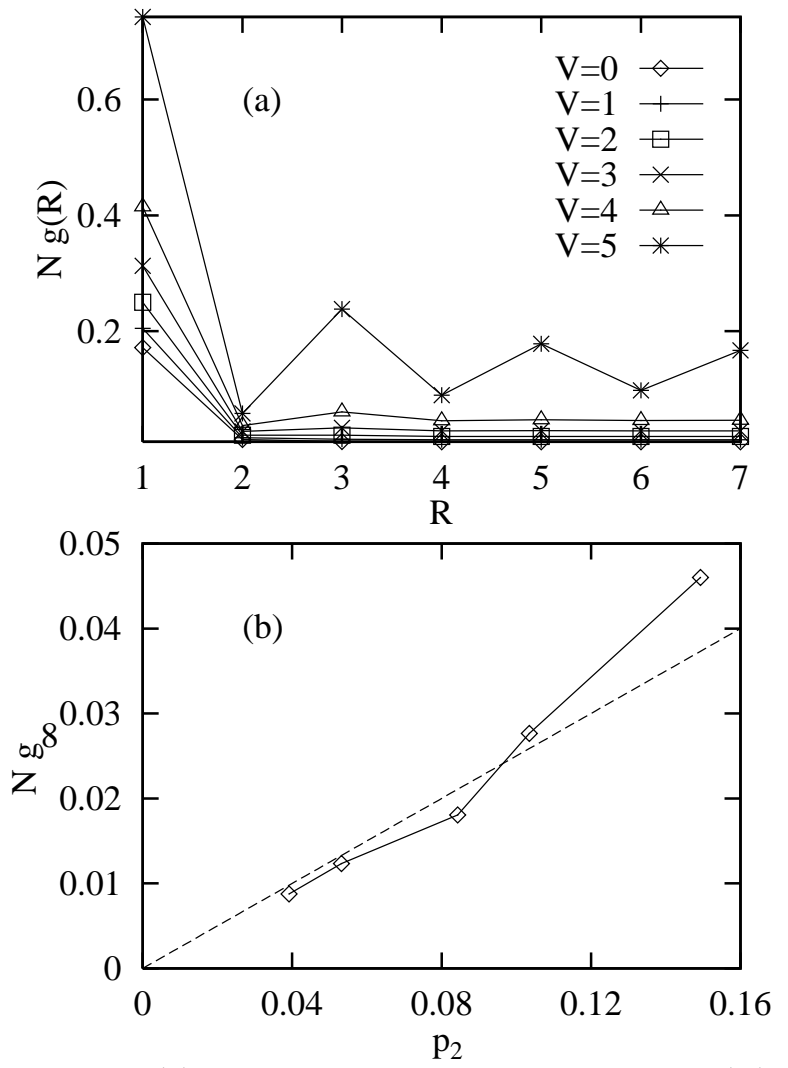

FIG. 2. (a) 'Empty-double correlation function' $g(R)$ in the ground state of a 14-site 1D Hubbard ring for different values of $V$. The other parameters are $U=10, t=1$.

(b) Asymptotic value $g_{\infty}$ vs. $p_{2}$, the probability to have two double occupancies in the ground state.

positions of the pairs are uncorrelated, this component has no more $R$-dependence. To check this interpretation, Fig. $2 \mathrm{~b}$ shows $g_{\infty}$ plotted versus $p_{2}$, the probability to have precisely two double occupancies in the system (for our relatively short chain and large value of $U$ the probability to have $n$ double occupancies decays exponentially with $n$, so that we may safely neglect corrections due to states with 3 or more double occupancies). To good approximation, $N \cdot g_{\infty}$ equals the probability to have more than one double occupancy in the chain, consistent with our interpretation. Assuming that a Heisenberg antiferromagnet with exchange constant $J=4 t^{2} /(U-V)$ describes the system well, we can moreover give a simple estimate of $g(R=1)$ : the number of doubly occupied sites is $\left\langle N_{d}\right\rangle=\partial_{U-V} E_{0}$, where $E_{0}=-N \cdot\left(4 t^{2} /(U-V)\right) \ln 2$ is the ground state energy of the Heisenberg antiferromagnet. Taking into account that on the average $50 \%$ of the double occupancies are to the right of 'their' hole we find $N g(R=1)=(1 / 2) \cdot\left\langle N_{d}\right\rangle=2 N(t /(U-V))^{2} \ln 2$. With the parameters used in Figure 2 we find e.g. $N g(R=1)=$ 
0.1664 for $V=0, N g(R=1)=0.6654$ for $V=5$ which values obviously provide reasonable estimates.

For large values of $V \approx U / 2$, i.e. the critical value for the onset of the CDW instability in 1D [5] we see in addition a modulation of $g(R)$ at longer distances with a period of two lattice spacings. This is obviously is a precursor of the CDW ordering which occurs for larger $V$. We may thus expect that $V=U / 2$ represents a limiting case, where the mapping to a nearest neighbor-exchange model starts to become inaccurate.

The empty-double correlation function $g(R)$ thus demonstrates that the main effect of $V$ is the enhancement of nearest neighbor charge fluctuations. This suggests that simple nearest neighbor Heisenberg exchange remains a good approximation also for the case $V>0$, the main difference as compared to the case $V=0$ being the enhancement of charge fluctuations and hence the Heisenberg exchange $J$. To check this we consider the dynamical spin correlation function $S(q, \omega)$, shown in Figure 3 a for different values of $V$.
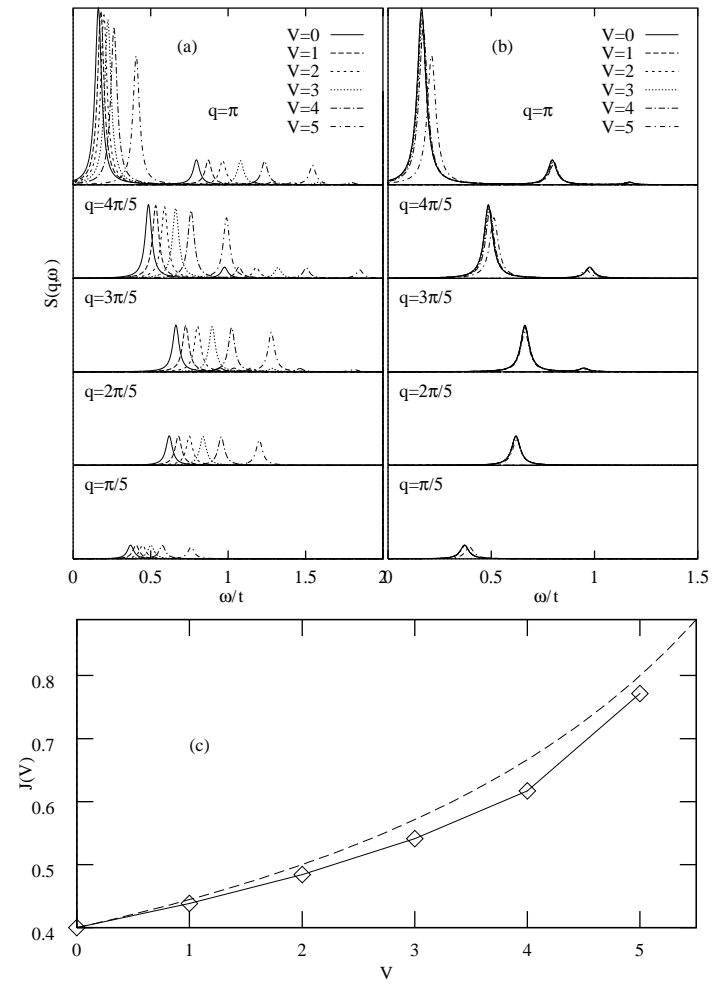

FIG. 3. (a) Spin correlation functions of a 10-site 1D Hubbard ring for different $V(U=10, t=1)$.

(b) Same as (a) but with the frequencies rescaled so as to make the energies of the dominant peak at $2 \pi / 5$ identical for all spectra.

(c) Effective Heisenberg exchange, $J_{e f f}(V)$, calculated from the rescaling factors used in (b), the dashed line shows $J_{\text {eff }}(V)=4 t^{2} /(U-V)$.

With increasing $V$, all peaks move to higher energy, the overall shape of the spectra being unaffected. Rescaling the frequencies the different $S(q, \omega)$ can be made practi- cally identical, as demonstrated in Figure 3 b. Assuming the relation $J=4 t^{2} / U$ to hold for $V=0$, we can use the rescaling factors to extract the effective Heisenberg exchange $J_{e f f}(V)$, shown in Figure 31c. Obviously values of $J_{e f f} / t$, as large as 0.8 , i.e. far outside the 'perturbational' range are possible. Figure 3 3 also shows the simple estimate $J_{\text {eff }}(V)=4 t^{2} /(U-V)$, which provides a reasonable approximation for the numerical values. Let us stress that for small values of $V$ this functional form of $J_{\text {eff }}(V)$ is rather trivial; what is not, however, is the validity of this relation up to the value $V=U / 2$.

We briefly turn to the charge transfer system. From Figure 1 we may expect that the $V$-term effectively increases the charge transfer energy $\Delta$ to $\Delta+V$ (it should be stressed, that this refers to 'bare' or 'model' parameters; in the case of the charge transfer insulator, the energy required to drag apart hole and double occupancy
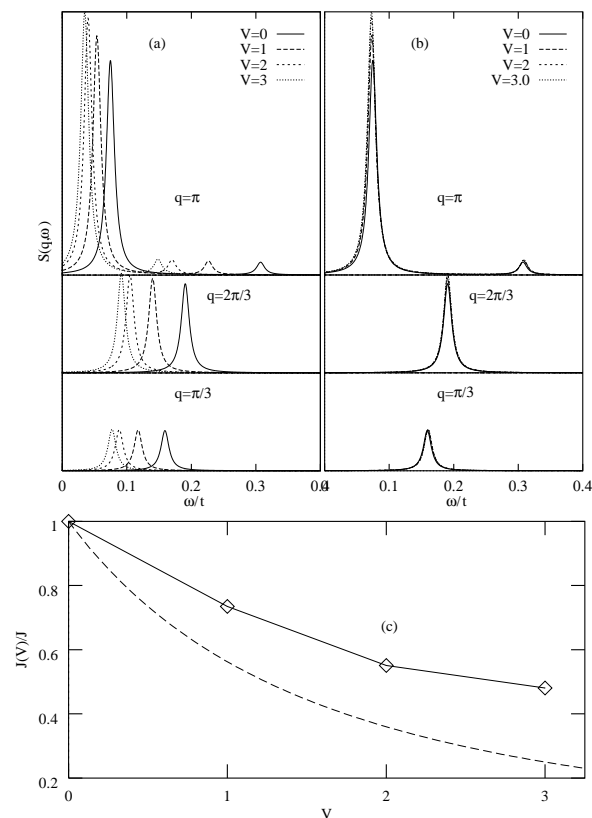

FIG. 4. (a) Spin correlation functions of a 6-unit cell $1 \mathrm{D}$ Charge transfer ring for different $V(\Delta=3, U=8, t=1)$.

(b) Same as (a) but with the frequencies rescaled so as to make the energies of the dominant peak at $2 \pi / 3$ identical for all spectra.

(c) Effective Heisenberg exchange, $J_{\text {eff }}(V)$, calculated from the rescaling factors used in (b). The dashed line has been calculated from $4^{\text {th }}$ order perturbation theory.

becomes $\min (\Delta+2 V, U)$ in the presence of the nearest neighbor repulsion, so that the experimentally measured charge transfer gap would be increased to $\Delta+2 \mathrm{~V}$ ). Contrary to the case of direct exchange, this suggests a suppression of the exchange constant $J$. This is demonstrated in Figure 4, which shows the dynamical spin correlation function for the charge transfer system with different values of $V$. From the rescaling of the SCF (see Figure $4 \mathrm{~b}$ ) we can again extract the effective exchange constant, which is shown in Figure Ac. The figure also 
shows a simple estimate based on $4^{\text {th }}$ order perturbation theory, where $J=\left(2 t_{p d} /(\Delta+V)\right)^{2} \cdot(1 / \Delta+1 / U)$, which however provides only a rough estimate.

We return to the single band model, and consider the doped case. In this case, the canonical transformation gives the strong-coupling model [2]:

$$
\begin{gathered}
H=-t \sum_{i, \sigma}\left(\hat{c}_{i, \sigma}^{\dagger} \hat{c}_{i+1, \sigma}+H . c .\right)+J \sum_{i}\left(\vec{S}_{i} \cdot \vec{S}_{j}-\frac{n_{i} n_{j}}{4}\right) \\
+\frac{J}{4} \sum_{i, \sigma}\left[\left(\hat{c}_{i, \sigma}^{\dagger} n_{i+1, \bar{\sigma}} \hat{c}_{i+2, \sigma}-\right.\right. \\
\left.\left.-\hat{c}_{i, \sigma}^{\dagger} \hat{c}_{i+1, \bar{\sigma}}^{\dagger} \hat{c}_{i+1, \sigma} \hat{c}_{i+2, \bar{\sigma}}\right)+H . c .\right]
\end{gathered}
$$

One may expect, that the projected model (2) is meaningful only for the description of the low energy part of the eigenvalue spectrum - at higher energy the extended

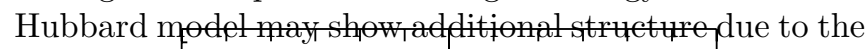

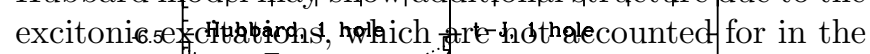
projected raopęl.

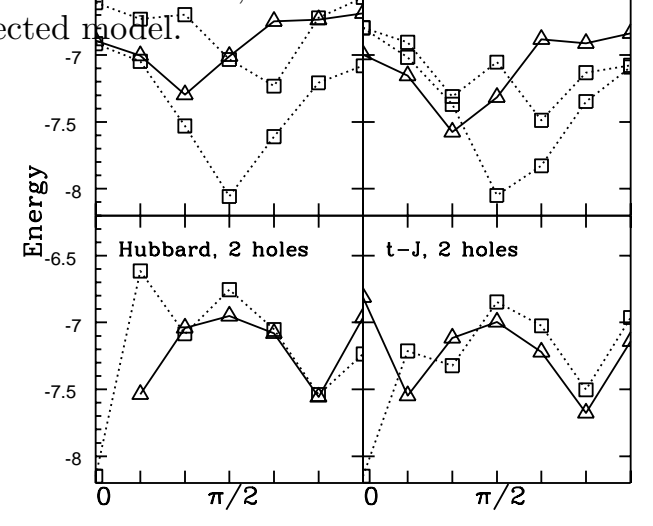

FIG. 5. Lowest eigenvalues for all allowed momenta of 12-site rings of extended Hubbard model $(U=10, V=5)$ and extended $t-J$ model $(J / t=0.8)$ for 11 and 10 electrons (i.e. one and two holes in the half-filled band). For the single hole, squares (triangles) denote states with $S=1 / 2(S=3 / 2)$, for two holes states with $S=0(S=1)$. States of like spin have been grouped into 'bands' only to guide the eye.

Figure 5 therefore compares the energies of the lowest eigenvalues of the extended Hubbard model with $U / V=2$, and the strong coupling model with $J / t=0.8$ (we have used the spin correlation function at half-filling to 'gauge' the value of $J$; moreover, a nearest neighbor density repulsion with $V=5$ has been added to the strong coupling model). While the agreement between the two level schemes is not really pefect, there is an obvious correspondence. Deviations are largest for the 2 hole states with $S=0$ near $\pi / 3=4 k_{F}$, which probably indicates a somewhat higher 'holon' velocity in the actual Hubbard model. This may be related to the fact that $U / V=2$ is at the very borderline of stability against formation of a CDW [5], and the empty-double correlation function showed already some signature of the CDW. Apart from that, the strong coupling model reproduces the overall energy scales of the Hubbard model quite well, which indicates that for low energy excitations, despite its apparently unphysically large exchange constant, the strong coupling model may indeed provide the appropriate 'effective' model.

In summary, we have investigated the influence of intersite Coulomb repulsion in the Hubbard model. For single band Hubbard models, our results suggest an enhancement of the exchange constant, roughly as $J(V)=$ $4 t^{2} /(U-V)$, whereas the spin dynamics still remains consistent with that of an ideal Heisenberg antiferromagnet. The remarkable feature of the results is that this rescaling seems to hold up to the CDW instability, which opens the way to unexpectedly large values of the ratio $J / t$. In particular, for the strong correlation case close to a CDW instability in $1 \mathrm{D}$, our results suggest values of $J$ comparable to the original hopping integral. Therefore, real materials which may be described by a strong correlation single band Hubbard model close to a CDW instability may provide realizations of $t-J$-like models with 'unphysically' large values of $J / t \approx 1$. For higher spatial dimensions, the critical value of $U / V$ for the formation of the CDW state is smaller than in $1 \mathrm{D}$, which seems to preclude large $J$ values due to inter-site repulsion. However, an alternative possibility here could be the suppression of the CDW due to a non-bipartite lattice structure, which can rule out the CDW even for large values of $V$; an interesting example here could be solid $C_{60}$, which crystallizes in the $f c c$ structure. Auger spectroscopy [6] gives values of $U \approx 1.5 \mathrm{eV}$, whereas analysis of the exciton dispersion [8] in undoped $C_{60}$ suggests values of $U-V$ as small as $0.35 \mathrm{eV}$, which is also consistent with other experimental estimates [7]. This is to be compared to the average bandwidth of the LUMO-derived bands of $\approx 0.3 \mathrm{eV}[9]$. While the inter-site Coulomb repulsion may be more efficiently screened in the doped material than the on-site $U$, leading to an increase of $U-V$, one still may expect relatively large values of $J$.

[1] A. B. Harris and R. V. Lange, Phys. Rev. 157, 295 (1967).

[2] K. A. Chao, J. Spalek, and A. M. Oles, J. Phys. C 58, L271 (1977).

[3] M. B. J. Meinders, L. H. Tjeng, and G. A. Sawatzky, Phys. Rev. Lett. 72, 2937 (1994).

[4] J. van den Brink et al., Phys. Rev. Lett. 75, 4658 (1995).

[5] J. E. Hirsch, Phys. Rev. Lett. 53, 2327 (1984).

[6] R.W. Lof, et al., Phys. Rev. Lett. 68, 3924 (1992).

[7] P.A. Brühwiler et al., Phys. Rev. Lett. 71, 3721 (1993);

[8] A.-M. Janner at al., Phys. Rev. B 52, 17158 (1995).

[9] S. Satpathy at al., Phys. Rev. B 46, 1773 (1992). 